\title{
A Novel Viscoelastic Material Modulus Function for Modifying the Golla-Hughes-McTavish Method
}

\author{
Luke A. Martin \\ Naval Surface Warfare Center Dahlgren Division, Dahlgren, Virginia, 22448, USA \\ Daniel J. Inman \\ Department of Aerospace Engineering, University of Michigan, Ann Arbor, Michigan, 48109, USA
}

(Received 28 September 2012; revised 29 November 2012; accepted 12 February 2013)

\begin{abstract}
The growing popularity of finite element analysis in the 1980s and 1990s spawned new techniques for modelling damping in complex structures. The Golla-Hughes-McTavish (GHM) method is one technique developed during this era. This method adds non-spatial degrees of freedom to a finite element model in order to account for a viscoelastic material's ability to dissipate energy. In the GHM method, a material modulus function is used to characterize the frequency-dependent complex modulus. This paper presents a novel material modulus function, thus modifying the GHM method. The advantages of this Modified Golla-Hughes-McTavish (MGHM) approach are a reduction in the curve fitting error and a standardized approach for computing material modulus coefficients. An additional parameter is introduced for each dissipation degree of freedom. This paper will compare the original GHM curve fit approach with this new standardized MGHM approach. Advantages of the MGHM approach and physical insight into the model are explained.
\end{abstract}

\section{INTRODUCTION}

The ability to accurately predict damping in structures is of primary importance to mechanical and civil engineers. In the age of computers, finite element analysis has become a popular tool for modelling structures ranging from automobiles and military aircrafts to bridges and buildings. The challenge when assembling a finite element model is how to incorporate damping in structures. When a structure is comprised of metals with few bolted joints, a lightly damped assumption can generally be made and applies to all the structural modes. However, structures using viscoelastic materials to passively control modes are more difficult to model because the lightly damped assumptions are no longer a reasonable estimate. Additionally, the viscoelastic material's damping properties vary as a function of frequency, causing the implementation for transient- and broadband-forcing functions to be more difficult. In the absence of reasonable prediction tools, the structural dynamicist often builds a prototype structure in order to experimentally determine the modal parameters. Most of today's finite element solvers do allow frequency-dependent material properties to be entered and can interpolate or extrapolate measured material modulus data within reason. A goal of this paper is to develop an accurate way to quantify material modulus functions and report these values as material properties. These dynamic material properties can then be used in simple multiple degrees of freedom simulations in MatLAB ${ }^{\circledR}$ or MathCAD ${ }^{\circledR}$. The same material properties are also appropriate for inclusion in higher fidelity finite element codes.

In 1985, Golla and Hughes introduced an approach to modelling viscoelastic damping, which can be implemented in finite element codes as a prediction tool. ${ }^{1}$ McTavish and Hughes expanded upon the Golla and Hughes work and defined the technique outlined as the Golla-Hughes-McTavish (GHM) method. ${ }^{2}$

In 1997, Friswell et al. introduced a four-parameter mini- oscillator term instead of the traditional three-parameter term. ${ }^{3}$ The motivation for the research presented in this article was taken from Friswell's search for the most generalized curve fit. Their paper also draws similarities between the GHM and Anelastic Displacement Field (ADF) ${ }^{4}$ methods, ultimately commenting that both methods introduce new augmented finite element coordinates as a way to account for how a viscoelastic material dissipates energy.

The past decade's literature does not contain additional works to further the GHM theory. The objective of this paper is to present a generalized MGHM theory that will allow researchers the advantage of having a set of equations with which to compute the MGHM coefficients. Note the GHM coefficients cannot be solved in closed form. This standard approach for computing MGHM coefficients could be adopted as a way to report material modulus functions for materials whose Young's modulus is a function of frequency. Furthermore, the MGHM method is advantageous over the ADF techniques because it casts the dynamic material modulus in terms of a material property. This approach is in contrast with the ADF technique that is built upon augmenting thermodynamic fields (ATFs). ATFs use the thermodynamic properties of materials to model the energy dissipated. This paper reviews the GHM theory and a modified GHM method using a novel material modulus function is presented. A standard mathematicallybased curve fitting approach for the modified GHM method is also introduced. Finally, a GHM and modified GHM curve fit for a commonly used viscoelastic material are compared. The first advantage of the MGHM method presented in this paper is that closed form solutions are derived for the MGHM parameters; previously, the GHM parameters could not be solved in closed form. An important note is that the parameters can now be solved, in general, for as many mini-oscillator terms as needed. The second advantage is that the curve fitting error of the MGHM model is reduced over the GHM model. 